\title{
Avaliação de um suplemento para animais idosos no tratamento de cães com sinais clínicos sugestivos da Síndrome da Disfunção Cognitiva Canina
}

\author{
[Evaluation of a supplement for elderly animals in the treatment of dogs with clinical signs \\ suggestive of Canine Cognitive Dysfunction Syndrome]
}

\section{"Artigo Científico/Scientific Article"}

\author{
Ariane Fortes Alfredo ${ }^{1}$, Dênis Antonio Ferrarin ${ }^{2}$, Risciela Salardi Alves de Brito ${ }^{3}$, Joice Lara \\ Maia Faria ${ }^{4}$, Amanda D'ávila Verardi ${ }^{4}$, Eduardo Negri Mueller ${ }^{4 *}$
}

\author{
${ }^{1}$ Médica Veterinária Autônoma, Chapecó-SC, Brasil. \\ ${ }^{2}$ Programa de Pós-Graduação em Medicina Veterinária, Universidade Federal de Santa Maria, Santa Maria-RS, Brasil \\ ${ }^{3}$ Programa de Pós-Graduação em Veterinária, Universidade Federal de Pelotas, Pelotas-RS, Brasil. \\ ${ }^{4}$ Instituto Federal Catarinense, Campus Concórdia, Concórdia-SC, Brasil. \\ *Autor para correspondência/Corresponding author: E-mail: eduardo.mueller@ifc.edu.br
}

\section{Resumo}

O alto nível de relação entre humanos e animais de companhia intensificou a preocupação de alguns tutores com as desordens relacionadas ao envelhecimento. Durante a velhice, além de mudanças fisiológicas, alterações patológicas como a Síndrome da Disfunção Cognitiva Canina (SDCC) são comuns. Essa doença é caracterizada por processos degenerativos que culminam em perda gradual da função cognitiva, sendo frequentemente confundida com o processo natural de envelhecimento. Neste contexto, objetivou-se avaliar a ação de um suplemento nutricional à base de aminoácidos, prebióticos e ácidos graxos para cães idosos na evolução clínica da SDCC. Foram avaliados 67 cães em idade sênior ( $\geq 7$ anos) para positividade aos sinais clínicos da SDCC. Posteriormente, os animais positivos $(n=15 / 22 \%)$ foram alocados em dois grupos experimentais, controle (C) e tratamento (T- utilização do suplemento nutricional). Observou-se melhora, relatada pelos tutores, de sinais clínicos associados à SDCC em $80 \%$ dos animais tratados, no entanto mais estudos são necessários para elucidar o efeito de suplementos nutricionais na regressão da sintomatologia clínica de cães com sinais clínicos sugestivos da doença.

Palavras-chave: envelhecimento; função cognitiva; nutrição.

\begin{abstract}
The high level of relationship between humans and pets has intensified the concern of some owners with disorders related to aging. As dogs age, in addition to physiological changes, pathological changes such as Canine Cognitive Dysfunction Syndrome (CCDS) are common. CCDS is characterized by a degenerative process that culminate in the gradual loss of cognitive function, being often confused with the natural process of aging. In this context, the main objective of this study was to evaluate the action of a nutritional supplement based on amino acids, prebiotics, and fatty acids on clinical evolution of CCDS in older dogs. Sixty-seven senior dogs ( $\geq 7$ years old) were evaluated for positivity of clinical signs of CCDS. Positive animals ( $\mathrm{n}=15$ / $22 \%$ ) were allocated into two experimental groups, control (C) and treatment (T- nutritional supplement). Owners reported improvement of clinical signs associated to CCDS in $89 \%$ of the therapy group, however, more studies are needed to elucidate the effect of nutritional supplements on the regression of clinical symptoms of dogs with clinical signs suggestive of the disease.
\end{abstract}

Keywords: ageing; cognitive function; nutrition. 


\section{Introdução}

A Síndrome da Disfunção Cognitiva Canina (SDCC) é uma doença neurodegenerativa progressiva que ocorre em cães idosos, sendo caracterizada por redução das funções cognitivas, tais como aprendizagem, memória, percepção e consciência, gerando comprometimento na manifestação do comportamento animal (Milgram et al., 1994). Nesta enfermidade ocorre deposição de placas de agregados moleculares extracelulares no cérebro, principalmente $\beta$-amilóide, semelhante ao que ocorre em humanos no Alzheimer (Borrás et al., 1999; Dimakopoulous e Mayer, 2002).

O acrônimo DISCA tem sido usado atualmente para descrever as categorias que sofrem alterações decorrentes da SDCC, sendo elas: desorientação, interação intra e interespécies, alterações no ciclo sono-vigília, perda do treino anterior aprendido em casa, e alteração nas atividades (Horwitz e Neilson, 2008).

O diagnóstico definitivo da doença é feito através do exame neuropatológico post mortem no cérebro. Porém, um diagnóstico presuntivo através de anamnese, testes neuropsicológicos e questionários específicos de alterações de comportamento, podem elucidar a ocorrência da doença nos cães senis (Svicero et al., 2017).

$\mathrm{O}$ tratamento da doença visa apenas o controle da síndrome. O primeiro medicamento utilizado para o tratamento da SDCC foi a selegilina, que é um inibidor seletivo e irreversível da monoamina-oxidase B (MAOB). A dieta é um tratamento preventivo eficaz, sua viabilidade foi testada durante dois anos através de testes neuropsicológicos. Além disto, enriquecimento ambiental, exercícios e novos comandos também favorecem a melhora da capacidade cognitiva (Landsberg, 2005). Dentre os tratamentos, os nutracêuticos e suplementos têm mostrado resultados satisfatórios na regressão dos sinais clínicos associados à SDCC (Osella et al., 2007). Porém, conforme já mencionado por Krug et al. (2017) ao revisar sobre o tema, ainda são necessários estudos no Brasil com relação à doença, diagnóstico e tratamentos farmacológicos. Neste contexto, objetivou-se avaliar a ação de um suplemento para cães idosos na evolução clínica do SDCC.

\section{Material e Métodos}

Foram incluídos neste estudo cães com idade superior a sete anos, de ambos os sexos, de diversas raças e portes, sem levar em conta estado reprodutivo do animal, atendidos no serviço de Clínica Médica do Instituto Federal Catarinense (IFC) - Campus Concórdia.

Como critérios de inclusão, foram aceitos cães que apresentavam alterações em duas ou mais das cinco categorias observadas de acordo com os sinais clínicos sugestivos da SDCC (Tabela 1), baseados na pesquisa de outros autores (Colle et al., 2000; Landsberg, 2005; Osella et al., 2007; Landsberg et al., 2012).

As cinco categorias incluíram desorientação, interação intra e interespécies, ciclo sono-vigília, perda do treino anterior aprendido em casa e atividades. Para uma categoria ser considerada alterada, dois itens dentro da mesma deveriam apresentar alterações. Os sinais deveriam ainda ter aparecido nesses animais a partir dos sete anos de idade ou terem se agravado a partir dessa idade. Pacientes que cumpriram estes requisitos foram então classificados como sugestivos a SDCC. O exame específico neurológico, assim como métodos de diagnóstico mais avançados, como ressonância magnética, tomografia computadorizada ou análise do líquido cérebroespinhal não foram realizados em nenhum paciente.

A amostra foi calculada a partir do levantamento dos prontuários arquivados no Centro de Práticas Clínicas e Cirúrgicas do IFC Campus Concórdia. Dos trezentos e sete cães atendidos até o mês de março de 2015, setenta apresentavam idade igual ou superior a sete anos. Os proprietários dos setenta cães foram convidados a colaborar com o trabalho, porém, sessenta e sete participaram da triagem. Dentre estes, apenas quinze cães apresentaram sinais clínicos sugestíveis da SDCC. Os dados foram apresentados na forma de distribuição de frequência e comparados quanto evolução do tratamento.

Os animais sugestivos a SDCC $(\mathrm{n}=15)$ foram classificados aleatoriamente em dois grupos. Os animais do grupo $\mathrm{T}$, tratamento $(\mathrm{n}=7)$ foram tratados com suplemento para cães idosos (Geripet ${ }^{\circledR}$ suplemento mineral vitamínico, empresa Vetnil Ind. e Com. De Produtos Veterinários Ltda., com sede na Avenida José Nicolau Stabile, nº53, Burck, Louveira - SP, Brasil) na dose recomendada pelo fabricante, de acordo com o peso do animal, e avaliados a cada trinta dias (Tabela 2). Esta avaliação foi elaborada a partir de pesquisas anteriores (Dewey, 2006; 
Landsberg e Head, 2008; Salvin et al., 2011), observados nos cães sugestivos a SDCC. contemplando os principais sinais clínicos

Tabela 1. Sinais clínicos de cães com diagnóstico sugestivo de Síndrome da Disfunção Cognitiva Canina (SDCC) avaliados no dia zero (D0).

\begin{tabular}{|c|c|c|}
\hline Sinais Clínicos & $\begin{array}{c}\text { Grupo T } \\
\mathbf{n}(\%) \\
\end{array}$ & $\begin{array}{c}\text { Grupo C } \\
\text { n(\%) } \\
\end{array}$ \\
\hline Desorientação & - & - \\
\hline 1. Perde-se em locais conhecidos & $1(16.6)$ & \\
\hline 2. Procura o lado errado da porta & - & - \\
\hline 3. Esbarra em objetos, não consegue transpor obstáculos & - & $1(14.2)$ \\
\hline 4. Olha fixo para o nada sem motivo aparente & $2(33.3)$ & $1(14.2)$ \\
\hline 5. Anda continuamente sem parar (repetitivamente e sem propósito) & - & - \\
\hline 6. Anda em círculos continuamente (repetitivamente e sem propósito) & - & - \\
\hline Total & $3(50)$ & $2(28,5)$ \\
\hline \multicolumn{3}{|l|}{ Ciclo sono/vigília } \\
\hline$\overline{7 .}$ Tem inquietação durante o sono & $3(50)$ & $1(14.2)$ \\
\hline 8. Acorda durante a noite e fica agitado & $2(33.3)$ & $2(28.5)$ \\
\hline 9. Late ou uiva durante a noite (não era seu hábito) & $3(50)$ & $1(14.2)$ \\
\hline 10. Dorme mais que o normal durante o dia & $5(83.3)$ & $4(57.1)$ \\
\hline Total & $6(100)$ & $5(71.4)$ \\
\hline \multicolumn{3}{|l|}{ Perda de Treinamento } \\
\hline 11. Urina/defeca em lugares desapropriados & $2(33.3)$ & $5(71.4)$ \\
\hline 12. Urina/defeca em seu lugar de descanso/dormir & $2(33.3)$ & $3(42.8)$ \\
\hline 13. Urina/defeca na frente de pessoas/proprietários & $1(16.6)$ & $3(42.8)$ \\
\hline 14. Esquece comandos já aprendidos & $2(33.3)$ & $2(28.5)$ \\
\hline 15. Incapaz de aprender novos comandos & $1(16.6)$ & $3(42.8)$ \\
\hline Total & $4(66.6)$ & $6(85.7)$ \\
\hline \multicolumn{3}{|l|}{ Interação } \\
\hline 16. Menor interesse por carinho/contato & $3(50)$ & $1(14.2)$ \\
\hline 17. Estranha pessoas conhecidas & $1(16.6)$ & $1(14.2)$ \\
\hline 18. Diminuição no comportamento de boas-vindas & $6(100)$ & $4(57.1)$ \\
\hline 19. Fica mais irritado e inquieto que o habitual & $2(33.3)$ & $3(42.8)$ \\
\hline 20. Recusa-se a passear, brincar & $1(16.6)$ & $4(57.1)$ \\
\hline 21. Não responde a comandos quando chamado & $3(50)$ & $4(57.1)$ \\
\hline 22. Late, chora mais que o costume & $5(83.3)$ & $2(28.5)$ \\
\hline 23. Segue outros animais & $2(33.3)$ & $3(42.8)$ \\
\hline 24. Demora em se acalmar depois de eventos estressantes & $4(66.6)$ & $4(57.1)$ \\
\hline \multirow{2}{*}{$\begin{array}{l}\text { 25. Comportamento agressivo para membros da família } \\
\text { Total }\end{array}$} & $1(16.6)$ & $2(28.5)$ \\
\hline & $6(100)$ & $6(85.7)$ \\
\hline \multicolumn{3}{|l|}{$\underline{\text { Atividade }}$} \\
\hline 26. Diminui a atividade de exploração & $3(33.3)$ & $2(28.5)$ \\
\hline 27. Aumento/Diminuição do apetite & $4(66.6)$ & $3(42.8)$ \\
\hline 28. Diminuição da capacidade de resposta a estímulos familiares & $4(66.6)$ & $2(28.5)$ \\
\hline \multirow{2}{*}{$\begin{array}{l}\text { 29. Mordisca o ar ou outros objetos } \\
\text { Total }\end{array}$} & $1(16.6)$ & $1(14.2)$ \\
\hline & $6(100)$ & $4(57.1)$ \\
\hline
\end{tabular}

*Grupo T (Tratamento) e grupo C (Controle)

Os animais enquadrados no grupo $\mathrm{C}(\mathrm{n}=8)$ receberam placebo em dose de acordo com o grupo $\mathrm{T}$ e também foram acompanhados a cada trinta dias. Todos os tutores foram instruídos quanto à administração dos comprimidos e à observação dos sinais clínicos sugestíveis da SDCC. O estudo foi conduzido da forma duplo-cego. Somente onze cães (5 do grupo T e 6 do grupo C) finalizaram noventa dias de tratamento.
A análise estatística foi realizada através do Teste de Kruskal-Wallis (5\%) após verificação da normalidade dos dados.

\section{Resultados}

Dois tutores decidiram não participar do estudo. Dos treze cães tratados, $8(61,53 \%)$ eram fêmeas (6 castradas e 2 inteiras) e $5(38,46 \%)$ machos (todos inteiros). A idade dos cães variou de 
oito a quinze anos (média 11,76 anos). As raças predominantes foram Pinscher ( $\mathrm{n}=3)$, Shih-Tzu, Poodle (ambos $\mathrm{n}=2$ ), Bichón Frise $(\mathrm{n}=1)$, Yorkshire $(n=1)$, Daschund $(n=1)$ e os demais $(n=3)$ sem raça definida (SRD). Dez $(76,92 \%)$ cães possuíam outros problemas de saúde, que não foram relacionados com o aparecimento dos sinais clínicos sugestivos a SDCC.

$\mathrm{Na}$ primeira avaliação (Tabela 1), previamente ao início dos tratamentos, predominaram alterações na interação e ciclo sono/vigília, seguidos dos sinais relacionados à atividade, perda de treinamento e desorientação.

Por opção do tutor, dois cães (um de cada grupo) foram excluídos do experimento aos doze dias de tratamento, sendo que no período não houve mudança nos sinais clínicos.

Tabela 2. Principais sinais clínicos avaliados em cães sugestivos de Síndrome da Disfunção Cognitiva Canina (SDCC) aos trinta dias de tratamento. Os animais do grupo $\mathrm{T}$ (Tratamento) foram tratados com suplemento para cães idosos e grupo C (Controle) com placebo. Os sinais clínicos foram avaliados quanto ao seu aumento ou diminuição de intensidade, observados pelos tutores. $\langle=$ menos; $>=$ mais.

\begin{tabular}{|c|c|c|c|c|c|c|}
\hline \multirow{2}{*}{ Sinais Clínicos (Dia 30) } & \multicolumn{3}{|c|}{ Grupo T n(\%) } & \multicolumn{3}{|c|}{ Grupo C n(\%) } \\
\hline & $<$ & Igual & $>$ & $<$ & Igual & $>$ \\
\hline $\begin{array}{l}\text { Comparado a } 1 \text { mês atrás, seu cão caminha em círculos e/ou } \\
\text { vagueia sem direção ou propósito? }\end{array}$ & - & $\begin{array}{c}5 \\
(100)\end{array}$ & - & - & $6(100)$ & - \\
\hline $\begin{array}{l}\text { Comparado a } 1 \text { mês atrás, seu cão olha fixamente para } \\
\text { paredes ou chão, sem propósito? }\end{array}$ & $1(20)$ & $4(80)$ & - & - & $6(100)$ & - \\
\hline $\begin{array}{l}\text { Comparado a } 1 \text { mês atrás, seu cão urina e/ou defeca em } \\
\text { áreas que não fazia antes? }\end{array}$ & $1(20)$ & $3(60)$ & $1(20)$ & - & $4(66,6)$ & $2(33,3)$ \\
\hline $\begin{array}{l}\text { Comparado a } 1 \text { mês atrás, seu cão falha em reconhecer } \\
\text { pessoas ou animais familiares? }\end{array}$ & - & $5(100)$ & - & $1(16.6)$ & $5(83,3)$ & - \\
\hline $\begin{array}{l}\text { Comparado a } 1 \text { mês atrás, seu cão responde a comandos } \\
\text { quando chamado? }\end{array}$ & - & $3(60)$ & $2(40)$ & - & $3(50)$ & $3(50)$ \\
\hline $\begin{array}{l}\text { Comparado a } 1 \text { mês atrás, seu cão late e/ou uiva mais que o } \\
\text { normal durante a noite? }\end{array}$ & $2(40)$ & $3(60)$ & - & - & $6(100)$ & - \\
\hline $\begin{array}{l}\text { Comparado a } 1 \text { mês atrás, seu cão demostra comportamento } \\
\text { de boas-vindas? }\end{array}$ & - & $2(40)$ & $3(60)$ & - & $4(66,6)$ & $2(33,3)$ \\
\hline $\begin{array}{l}\text { Comparado a } 1 \text { mês atrás, seu cão tem interesse por } \\
\text { carinho/contato? }\end{array}$ & - & $4(80)$ & $1(20)$ & - & $5(83,3)$ & $1(16,6)$ \\
\hline $\begin{array}{l}\text { Comparado a } 1 \text { mês atrás, seu cão esbarra em } \\
\text { paredes/portas/objetos? }\end{array}$ & - & $5(100)$ & - & - & $6(100)$ & - \\
\hline $\begin{array}{l}\text { Comparado a } 1 \text { mês atrás, seu cão se recusa a brincar ou } \\
\text { passear? }\end{array}$ & - & $5(100)$ & - & - & $6(100)$ & - \\
\hline
\end{tabular}

Dos 5 animais do grupo $\mathrm{T}$ que finalizaram os noventa dias de tratamento, 4 (80\%) cães, segundo os tutores apresentaram melhora nos sinais clínicos, e apenas em 1 (20\%) cão os sinais clínicos permaneceram iguais. Os sinais clínicos descritos pelos tutores que demonstraram melhores resultados foram aumento no comportamento de boas-vindas, maior resposta a comandos quando chamado e diminuição do latir e/ou uivar mais que o normal durante a noite. Dos 6 cães do grupo C, 2 não apresentaram melhora clínica e 2 deles urinam e/ou defecam com mais intensidade em áreas que não faziam antes, outras alterações estão listadas na Tabela 2. Através da análise estatística verificou-se que para nenhum dos comportamentos estudados houve diferença estatística entre os grupos controle e tratamento.

\section{Discussão}

O uso de um suplemento vitamínico mineral foi investigado e comparado com um grupo controle (placebo) quanto a sua capacidade de alteração na evolução dos sinais clínicos sugestíveis da SDCC. Dos animais que receberam o suplemento, $80 \%$ deles apresentaram diminuição dos sinais clínicos, associados à SDCC, segundo respostas dos tutores. O uso de nutracêuticos, especialmente os antioxidantes poderiam ser considerados para o tratamento da disfunção cognitiva, embora com dificuldade de determinar dosagem, efeitos a longo prazo, entre outros fatores (Head e Zicker, 2004).

A prevalência de cães que apresentavam sinais clínicos sugestivos da SDCC no presente estudo $(22,38 \%$ - 15/67) está dentro dos valores encontrados por Azkona et al. (2009) de 22,5\%, Heath et al. (2007) de $20-30 \%$ e por Bain et al. (2001) de $22 \%$ em cães com idade entre 11 e 14 anos. Quanto maior a idade dos cães, mais alterações comportamentais (Neilson et al., 2001), visto que os idosos demonstram um declínio cognitivo dependente da idade e de afecções 
cerebrais existentes (Faraco, 2013). Quanto ao sexo, já foi observado que cães machos apresentaram um fator de risco maior para alterações comportamentais do que fêmeas, embora não tenha sido considerado o status reprodutivo (Svicero et al., 2017). A categoria mais afetada, assim como no estudo de Osella et al. (2007) foi a de interação, principalmente a redução do comportamento de boas-vindas.

No estudo, seis cães apresentavam alterações em duas categorias do acrônimo DISCA, seis cães em três categorias e 1 cão apresentou alterações em quatro categorias. Embora o questionário DISCA seja abrangente, outros sinais menos frequentes podem existir (Landsberg, 2005). A existência de alterações não significa que o animal apresente necessariamente a SDCC, pois o aparecimento dos sinais pode estar associado a outras doenças. Portanto, seu diagnóstico é feito através da exclusão de outras afecções que cursem com a mesma sintomatologia. O presente estudo não incluiu dados referentes ao exame neurológico ou de exames como ressonância magnética, tomografia computadorizada ou análise de líquido cérebro-espinhal, pois mimetiza a realidade de pequenos centros urbanos e unidades de cuidado veterinário primário, onde tais recursos ou competências nem sempre são disponíveis. Outros estudos abrangendo sinais clínicos compatíveis com a SDCC já foram publicados sem a divulgação de dados sobre o exame neurológico ou outros auxílios ao diagnóstico, baseados somente em questionário comportamental (Krug et al., 2019). Landsberg (2005) também ressalta a convivência e interação entre humanos e animais como a principal maneira de diagnosticar a SDCC.

Pesquisas confirmam que cães com sinais clínicos sugestivos de SDCC podem apresentar melhora dos sinais clínicos se enriquecida dieta com suplemento alimentar (Landsberg, 2005; Heath et al., 2007). Constituintes do produto comercial utilizado incluem vitaminas, como a vitamina A, C, E, Beta-caroteno (sequestram os radicais livres) e taurina, os quais exercem uma função protetora contra as doenças neurodegenerativas (Bianchi e Antunes, 1999; Heaton et al., 2002). Seu uso é capaz de retardar o déficit cognitivo, bem como as alterações comportamentais associadas à SDCC (Landsberg, 2005). Proteínas e uma gama de antioxidantes que podem ajudar no retardo da doença também fazem parte da constituição do produto. A glutamina é um aminoácido livre, sendo classificada como aminoácido não essencial para monogástricos, porém está diretamente relacionada às funções metabólicas, assim como a L-carnitina considerado um nutriente não essencial, mas que desenvolve papel importante na produção de energia celular (Lacey e Wilmore, 1990; Rodrigues et al., 2003). O inositol, componente importante do suplemento vem sendo estudado e sabe-se que ele desempenha papel importante na regressão dos sinais clínicos da doença de Alzheimer, bem como na SDCC. Acredita-se que ele possa ter um efeito antiagregação de amilóide, inibindo déficits cognitivos (Felini et al., 2007).

Grande importância deve ser dada também às alterações metabólicas. Sabe-se que o estresse oxidativo contribui de forma significante para o processo da neurodegeneração, reduzindo antioxidantes endógenos, diminuindo a produção cerebral de melatonina e secreção de neurotransmissores excitatórios do Sistema Nervoso Central (SNC) importantes na ativação da memória, como a noradrenalina e acetilcolina (Cotman et al., 2002; Teixeira, 2009).

A aparente melhora dos três cães do grupo $\mathrm{C}$ pode estar relacionada com o chamado efeito ou resposta placebo, que segundo Teixeira (2009) é a melhora dos sinais clínicos ou funções fisiológicas do organismo em resposta a fatores inespecíficos e aparentemente inertes (comprimido placebo), sendo atribuída ao simbolismo que o tratamento exerce na expectativa positiva do paciente, neste caso, na expectativa dos tutores. Também pode estar associada a melhor observação por parte dos tutores após início do tratamento. Dentre os animais do grupo $\mathrm{C}$, dois deles urinam e/ou defecam fora de lugares apropriados com maior frequência.

Uma série de testes neuropsicológicos relacionados à capacidade de aprendizagem e memória podem ser realizados em laboratórios, eles mensuram o declínio ou melhora das funções cognitivas em cães com sinais clínicos da SDCC (Landsberg, 2005), porém sua aplicabilidade na rotina clínica é baixa. Portanto, o que se tornou viável para aplicar ao tutor foi o questionário específico para determinadas alterações de comportamentos que são sugestivas à doença. Futuras pesquisas podem obter melhores resultados, se realizadas em ambiente de internação com avaliações e acompanhamento constante de pessoas treinadas e capacitadas.

Como houve tendência na diminuição dos sinais clínicos associados à SDCC em cães tratados 
durante trinta dias, o uso de suplemento alimentar por tempo prolongado pode diminuir de forma mais acentuada os sinais clínicos sugestivos de SDCC, como relatado nos estudos de Landsberg (2005) e Heath et al. (2007). Portanto, o tempo de pesquisa deve ser prolongado para que melhores resultados sejam observados, podendo auxiliar em futuros tratamentos da SDCC.

Os tutores mostraram-se interessados em continuar com a suplementação dos animais, relataram facilidade na administração dos comprimidos e houve aumento de apetite em todos os cães do grupo tratados com suplemento, podendo a avaliação de ganho de peso estar presente em futuros estudos.

\section{Conclusão}

A possível diminuição na melhora dos sinais clínicos associados à SDCC, relatada pelos tutores, principalmente na categoria de interação social, sugere que $\mathrm{o}$ enriquecimento da dieta com suplemento para idosos pode ser útil na regressão da sintomatologia clínica de cães com sinais clínicos sugestivos da SDCC.

\section{Conflitos de interesse}

Os autores declaram não existir conflito de interesse.

\section{Comitê de Ética}

$\mathrm{O}$ projeto de pesquisa foi aprovado pelo Comitê de Ética em Uso de Animais do IFCCampus Concórdia sob n ${ }^{\circ}$ 25/2014.

\section{Referências}

Azkona, G.; Garcia-Belenguer, S.; Chacón, G.; Rosado, B.; Leon, M.; Palacio, J. Prevalence and risk factors of behavioral changes associated with age-related cognitive impairment in geriatric dogs. Journal of Small Animal Practice, 50(2): 87-91, 2009.

Bain, M.J.; Hart, B.L.; Cliff, K.D.; Ruehl, W.W. Predicting behavioral changes associated with age-related cognitive impairment in dogs. Journal of American Veterinary Medical Association, 218(11): 1792-1795, 2001.

Bianchi, M.L.P.; Antunes, L.M.G. Radicais livres e os principais antioxidantes da dieta. Revista de Nutrição, 12(2): 123-130, 1999.

Borrás, D.; Ferrer, I.; Pumarola, M. Age-related changes in the brain of the dog. Veterinary Pathology, 36(3): 202-211, 1999.

Colle, M.A.; Hauw, J.J.; Crespeau, F.; Uchihara,
T.; Akiyama, H.; Checler, F.; Pageat, P.; Duykaerts, C. Vascular and parenchymal AB deposition in the aging dog: correlation with behavior. Neurobiology of Aging, 21(5): 695-704, 2000.

Cotman, C.W.; Head, E.; Muggenburg, B.A.; Zicker, S.; Milgram, N.W. Brain aging in the canine: a diet enriched in antioxidants reduces cognitive dysfunction. Neurobiology of Aging, 23(5): 809-818, 2002.

Dewey, C.W. Neurologia de cães e gatos - guia prático. $1^{\text {a }}$ ed. São Paulo: Roca, 2006. 368p.

Dimakopoulous, A.C.; Mayer, R.J. Aspects of neurodegeneration in the canine brain. The Journal of Nutrition, 132(6): 1579-1582, 2002.

Faraco, C.B. Envelhecimento dos cães e gatos. In: Faraco, C.B.; Soares, G.M. Fundamentos do comportamento canino e felino. São Paulo: MedVet Ltda, 2013. p. 187-193.

Felini, D.; Brown, M.; Rappaport, R.; Mclaurim, J. Properties of scyllo-inositol as a therapeutic treatment of AD-like pathology. Journal of Molecular Medicine, 85(6): 603-611, 2007.

Head, E.; Zicker, S.C. Nutraceuticals, aging, and cognitive dysfunction. Veterinary Clinics: Small Animal Practice, 34(1): 217-228, 2004.

Heath, S.E.; Barabas, S.; Craze, P.G. Nutricional supplementation in cases of canine cognitive dysfunction - A clinical trial. Applied Animal Behaviour Science, 105(4): 284-296, 2007.

Heaton, P.R.; Reed, C.F.; Mann, S.J.; Ransley, R.; Stevenson, J.; Charlton, C.J. Rawlings, J.M. Role of Dietary Antioxidants to Protect against DNA Damage in Adult Dogs. The Journal of Nutrition, 132(6): 720-1724, 2002.

Horwitz, D.F.; Neilson, J.C. Comportamento canino \& felino. $1^{a}$ ed. Porto Alegre: Artmed, 2008. 662p.

Krug, F.D.M.; Lima, C.S.; Tillmann, M.T.; Nobre, M.O. Síndrome da disfunção cognitiva canina. Medvep - Revista Científica de Medicina Veterinária - Pequenos Animais e Animais de Estimação, 15(46): 106-110, 2017.

Krug, F.D.M.; Tillmann, M.T.; Piñero, M.B.V.; Capellas, S.D.O.; Costa, A.L; Bruhn, F.R.P.; Nobre, M.D.O. Evaluation of cognitive dysfunction syndrome in dogs using an observational questionnaire. Semina: Ciências Agrárias, 40(5, sup. 1): 2235-2246, 2019. 
Lacey, J.M.; Wilmore, D.W. Is glutamine a conditionally essential amino acid? Nutrition Reviews, 48(8): 297-309, 1990.

Landsberg, G. Therapeutic agents for the treatment of cognitive dysfunction syndrome in senior dogs. Progress in NeuroPsychopharmacology and Biological Psychiatry, 29(3): 471-479, 2005.

Landsberg, G.M.; Head, E. Senilidade e seus efeitos sobre o comportamento. In: Hoskins, J.D. Geriatria e Gerontologia do Cão e Gato. $2^{\text {a }}$ ed. São Paulo: Roca, 2008. p.33-48.

Landsberg, G.M.; Nichol, J.; Araujo, J. Cognitive Dysfunction Syndrome A Disease of Canine and Feline Brain Aging. Veterinary Clinics of North America: Small Animal Practice, 42: 749-768, 2012.

Milgram, N.W.; Head, E.; Weiner, E.; Thomas, E. Cognitive functions and aging in the dog: acquisition of non-spatial visual tasks. Behavioral Neuroscience, 108(1): 57-68, 1994.

Neilson, J.C.; Hart, B.L.; Cliff, K.D.; Ruehl, W.W. Prevalence of behavioral changes associated with age-related cognitive impairment in dogs. Journal of American Veterinary Association, 218(11): 1787-1791, 2001.
Osella, M.C.; Odore, R.; Girardi, C.; Badino, P.; Barbero, R.; Bergamasco, L. Canine cognitive dysfunction syndrome: Prevalence, clinical signs and treatment with a neuroprotective nutraceutical. Applied Animal Behaviour Science, 105(4): 297-310, 2007.

Rodrigues, L.P.; Padovan, G.J.; Marchini, J.S. Uso da carnitina em terapia nutricional. Nutrire: Revista da Sociedade Brasileira de Alimentação e Nutrição, 25: 113-134, 2003. Salvin, H.E.; Mcgreevy, P.D.; Sachdev, P.S.; Valenzuela, M.J. The canine cognitive dysfunction rating scale (CCDR): A datadriven and ecologically relevant assessment tool. The Veterinary Journal, 188(3): 331336, 2011.

Svicero, D.J.; Heckler, M.C.T.; Amorim, R.M. Prevalence of behavioral changes in senile dogs. Ciência Rural, 47(2): e20151645, 2017.

Teixeira, M.Z. Bases psiconeurofisiológicas do fenômeno placebo-nocebo: evidências científicas que valorizam a humanização da relação médico-paciente. Revista da Associação Médica Brasileira, 55(1): 13-18, 2009. 